\title{
Towards Counterexample-guided k-Induction for Fast Bug Detection
}

DOI:

$10.1145 / 3236024.3264840$

\section{Document Version}

Accepted author manuscript

Link to publication record in Manchester Research Explorer

\section{Citation for published version (APA):}

Gadelha, M. Y. R., Monteiro, F. R., Cordeiro, L., \& Nicole, D. A. (2018). Towards Counterexample-guided kInduction for Fast Bug Detection. In 25th ACM SIGSOFT International Symposium on the Foundations of Software Engineering https://doi.org/10.1145/3236024.3264840

\section{Published in:}

25th ACM SIGSOFT International Symposium on the Foundations of Software Engineering

\section{Citing this paper}

Please note that where the full-text provided on Manchester Research Explorer is the Author Accepted Manuscript or Proof version this may differ from the final Published version. If citing, it is advised that you check and use the publisher's definitive version.

\section{General rights}

Copyright and moral rights for the publications made accessible in the Research Explorer are retained by the authors and/or other copyright owners and it is a condition of accessing publications that users recognise and abide by the legal requirements associated with these rights.

\section{Takedown policy}

If you believe that this document breaches copyright please refer to the University of Manchester's Takedown Procedures [http://man.ac.uk/04Y6Bo] or contact uml.scholarlycommunications@manchester.ac.uk providing relevant details, so we can investigate your claim.

\section{OPEN ACCESS}




\section{Towards Counterexample-guided $\boldsymbol{k}$-Induction for Fast Bug Detection}

\author{
Mikhail Y. R. Gadelha \\ University of Southampton \\ United Kingdom \\ myrg1g14@soton.ac.uk \\ Lucas C. Cordeiro \\ University of Manchester \\ United Kingdom \\ lucas.cordeiro@manchester.ac.uk
}

\author{
Felipe R. Monteiro \\ Motorola Mobility, A Lenovo Company \\ Brazil \\ frsousa@motorola.com \\ Denis A. Nicole \\ University of Southampton \\ United Kingdom \\ dan@ecs.soton.ac.uk
}

\begin{abstract}
Recently, the $k$-induction algorithm has proven to be a successful approach for both finding bugs and proving correctness. However, since the algorithm is an incremental approach, it might waste resources trying to prove incorrect programs. In this paper, we propose to extend the $k$-induction algorithm in order to shorten the number of steps required to find a property violation. We convert the algorithm into a meet-in-the-middle bidirectional search algorithm, using the counterexample produced from overapproximating the program. The preliminary results show that the number of steps required to find a property violation is reduced to $\left\lfloor\frac{k}{2}+1\right\rfloor$ and the verification time for programs with large state space is also reduced considerably.
\end{abstract}

\section{CCS CONCEPTS}

- Hardware $\rightarrow$ Bug detection, localization and diagnosis; • Software and its engineering $\rightarrow$ Formal software verification; • Theory of computation $\rightarrow$ Verification by model checking;

\section{KEYWORDS}

Bounded Model Checking; $k$-induction; Formal Software Verification; Bug detection.

\section{ACM Reference Format:}

Mikhail Y. R. Gadelha, Felipe R. Monteiro, Lucas C. Cordeiro, and Denis A. Nicole. 2018. Towards Counterexample-guided $k$-Induction for Fast Bug Detection. In Proceedings of The $26^{\text {th }}$ ACM Joint European Software Engineering Conference and Symposium on the Foundations of Software Engineering (ESEC/FSE 2018). ACM, New York, NY, USA, 5 pages. https: //doi.org/10.1145/nnnnnnn.nnnnnnn

\section{INTRODUCTION}

Embedded systems are used in a variety of applications, ranging from nuclear plants and automotive systems to entertainment and games [9]. This ubiquity drives a need to test and validate a system

Permission to make digital or hard copies of part or all of this work for personal or classroom use is granted without fee provided that copies are not made or distributed for profit or commercial advantage and that copies bear this notice and the full citation on the first page. Copyrights for third-party components of this work must be honored.

For all other uses, contact the owner/author(s).

ESEC/FSE 2018, 4 - 9 Nov, 2018, Lake Buena Vista, Florida, USA

(C) 2018 Copyright held by the owner/author(s)

ACM ISBN 978-x-xxxx-xxxx-x/YY/MM.

https://doi.org/10.1145/nnnnnnn.nnnnnnn before releasing it to the market, in order to protect against system failures. Even subtle system bugs can have drastic consequences, such as the recent Heartbleed bug on OpenSSH, which might have leaked private information from several servers [7].

One promising technique to verify embedded software is called bounded model checking (BMC) [3]. The basic idea of BMC is to check the negation of a property at a given depth: given a transition system $M$, a property $\phi$, and a bound $k$, BMC unrolls the system $k$ times and generates verification conditions (VC) $\psi$, such that $\psi$ is satisfiable iff $\phi$ has a counterexample of depth $k$ or less. BMC tools based on Boolean Satisfiability (SAT) or Satisfiability Module Theories (SMT) have been applied on the verification of both sequential and parallel programs $[5,6,14,15]$. However, BMC tools are aimed to find bugs; they cannot prove correctness, unless the bound $k$ safely reaches all program states [8].

Despite the fact that BMC cannot prove correctness by itself (unless it fully unwinds the program), there are algorithms that use BMC as a "component" to prove correctness. In particular, the $k$-induction algorithm is an incremental approach that aims to find bugs and prove correctness using an ever increasing number of unwindings. In this paper, we propose to extend the algorithm originally developed for $k$-induction to shorten the number of iterations required to find a property violation. Our main original contribution is an extension to the $k$-induction algorithm, which converts the algorithm into a meet-in-the-middle bidirectional search by using the counterexample generated by the inductive step (c.f., Section 3 ). In fact, the preliminary results show that the number of steps required to find a property violation is reduced to $\left\lfloor\frac{k}{2}+1\right\rfloor$ and the verification time for programs with large state space is reduced considerably (c.f., Section 4).

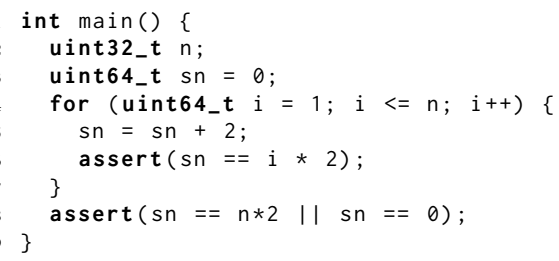

Figure 1: ANSI-C program example with an upper-bound limit up to $2^{32}-1$. 


\section{THE $K$-INDUCTION ALGORITHM}

The first version of the $k$-induction algorithm was proposed by Sheeran et al. [16]; they apply BMC to find bugs and prove correctness. BMC tools cannot prove correctness unless the bound $k$ is appropriate to reach the completeness threshold (i.e., a value that will fully unroll all loops occurring in the program, often impractically large) [11]. For instance, consider the simple program shown in Figure 1, the assertion in line 8 always

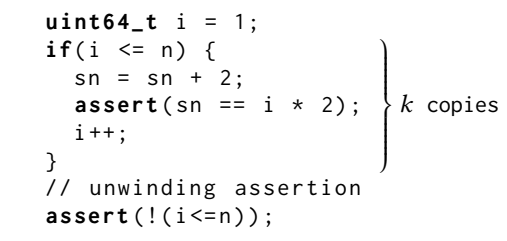

Figure 2: Finite $k$ unwindings done by BMC. holds, regardless of the initial value of $\mathrm{n}$ in line 2. BMC tools as CBMC [5], ESBMC [6] or LLBMC [13] typically reproduce the loop (lines $4-7$ in Figure 1) as the code snippet in Figure 2 and are unable to verify that program unless the loop is fully unrolled, i.e., the unwinding assertion if $k<2^{32}-1$ in 32-bit and 64-bit architectures.

Consider that $T\left(s_{i}, s_{i+1}\right)$ is the transition relations for $m$ over the state variables $s_{i}$ and $s_{i+1}$, $\Phi$ is the set of safety properties, $\phi(s) \in \Phi$ is the formula encoding for states satisfying a safety property, and $\psi(s)$ is the formula encoding for states satisfying a completeness threshold [12], which can be smaller than or equal to the maximum number of loop-iterations occurring in the program. Based on such formalization, the $k$-induction algorithm performs three checks for each step $k$ : the base case $B_{k}(k)$, forward condition $F_{k}(k)$ and inductive step $I_{k}(k)$, for $k=[1, d]$, where $d$ is the depth of the transition system $m$ [8]. In the first check, the base case $B_{k}(k)$ is the standard BMC and it is satisfiable iff $B_{k}(k)$ has a counterexample of length $k$ or less [2]:

$$
B_{k}(k)=\exists s_{1} \ldots s_{k} \cdot I\left(s_{1}\right) \wedge \bigwedge_{i=1}^{k-1} T\left(s_{i}, s_{i+1}\right) \wedge \bigvee_{i=1}^{k} \neg \phi\left(s_{i}\right) .
$$

In the second check, the forward condition $F_{k}(k)$ checks if the completeness threshold $\psi(s)$ holds for the current $k$. This is established by checking if the following is unsatisfiable:

$$
F_{k}(k)=\exists s_{1} \ldots s_{k} . I\left(s_{1}\right) \wedge \bigwedge_{i=1}^{k-1} T\left(s_{i}, s_{i+1}\right) \wedge \bigvee_{i=1}^{k} \neg \psi\left(s_{k}\right) .
$$

No safety property $\phi(s)$ is checked in $F_{k}(k)$ as they were already checked for the current $k$ in the base case. Finally, the inductive step $I_{k}(k)$, checks if whenever $\phi(s)$ holds in $k$ states $s_{1}, \ldots, s_{k}, \phi(s)$ also holds for the next state $s_{k+1}$. This is established by checking if the following is unsatisfiable:

$$
I_{k}(k)=\exists s_{1} \ldots s_{k+1} \cdot \bigwedge_{i=1}^{k}\left(\phi\left(s_{i}\right) \wedge T\left(s_{i}, s_{i+1}\right)\right) \wedge \neg \phi\left(s_{k+1}\right) .
$$

Therefore, the $k$-induction algorithm at a given $k$ is:

$$
k_{\text {ind }}(P, k)= \begin{cases}P \text { has a bug, } & \text { if } \neg B_{k}(k), \\ P \text { is correct, } & \text { if } B_{k}(k) \wedge\left[F_{k}(k) \vee I_{k}(k)\right], \\ k_{\text {ind }}(P, k+1), & \text { otherwise. }\end{cases}
$$

It worth noticed in Eq. (4) that a bug is only reported in the base case (i.e., $\left.B_{k}(k)\right)$ and if a violation is reported in the inductive step (i.e., $\left.I_{k}(k)\right)$ the algorithm assumes the results is spurious, thus, it calls itself recursively for the next iteration. The $k$-induction algorithm is a complete and optimal search algorithm (i.e., always find the shortest counterexample), with complexity $O(b d)$ and state space $O\left(b^{+} d^{+}\right)$[8]. Indeed, Jovanović et al. [10] prove that the $k$ induction proof rule can be more powerful and concise than regular induction.

\section{COUNTEREXAMPLE-GUIDED $K$-INDUCTION ALGORITHM}

The $k$-induction algorithm is being applied to solve a number of different verification problems, but it has its own limitations. The biggest one is the fact that if a state $\xi$ violates a property, the algorithm requires $k$ steps to find the counterexample. This is expensive because of the three checks performed for each $k$. The inductive step $I_{k}(k)$ is the most computationally expensive one; it is an overapproximation, forcing the SMT solver to find a set of assignments in a larger state space than the original program [8]. Moreover, the computation is wasted if a counterexample is found by the inductive step, as it is assumed to be spurious (c.f. Section 2).

Consider a program $P$ that contains a set of variables $V=$ $\left\{v_{1}, \ldots, v_{n}\right\}$, where $n$ is the number of variables in the program, and its control-flow graph (CFG) that represents an state transition system. In the CFG, an state $s_{i}$ is a tuple $\left\langle p_{c}, V_{i}\right\rangle$, where $p_{c}$ is the program counter and $V_{i}=\left\{v_{1}^{i}, \ldots, v_{n}^{i}\right\}$ are the values of all program variables. A transition $t$ is a guarded assignment $\langle[\gamma], x:=$ e), where $\gamma$ is a predicate over the program variables and $e$ is an expression assigned to $\mathrm{x}$.

Definition 1. Counterexample is a sequence of states $\pi=$ $\left\langle s_{i}, \ldots, \xi\right\rangle$ of length $k$ that represents a path from an initial state $s_{i}$ to an error state $\xi$.

In order to tackle the aforementioned problems in the $k$-induction algorithm, we propose to use the counterexample generated by the inductive step, to speed up the bug finding check (i.e., the base case). Our extension aims to convert the $k$-induction algorithm into a bidirectional search approach, by search simultaneously both forward (i.e., from the initial state $s_{1}$ ) and backward (i.e., from the error state $\xi$ detected in the inductive step $\left.I_{k}(k)\right)$ and stop if the both searches meet in the middle as shown in Figure 3.

The base case $B_{k}(k)$ is the forward part of the algorithm, since it tries to find a counterexample $\pi_{B}=\left\langle s_{1}, \ldots, \xi\right\rangle$ that represents a path from the initial state of the program $\mathrm{P}\left(\right.$ i.e., $\left.s_{1}\right)$ to an error state $\xi$. The inductive step $I_{k}(k)$ is the backward part of the algorithm; it tries to find a counterexample $\pi_{I}=\left\langle s_{d}, \ldots, \xi\right\rangle$, from any depth $d$ in the CFG.

LEMmA 1. The counterexample $\pi_{I}=\left\langle s_{d}, \ldots, \xi\right\rangle$ produced by the inductive step $I_{k}(k)$ is a path that leads to a property violation (i.e., an error state $\xi$ ); reaching any value in that path (i.e., $s_{d}, s_{d+1}, \ldots$, $\xi)$ will lead to this property violation.

Based on Lemma 1, if at least one state of $\pi_{I}\left(\right.$ e.g., $\left.s_{i}\right)$ is reachable from the initial state $s_{1}$, then the error state $\xi$ is reachable from the initial state $s_{1}$. Thus, given a counterexample $\pi_{I}=\left\langle s_{i}, \ldots, \xi\right\rangle$ from 

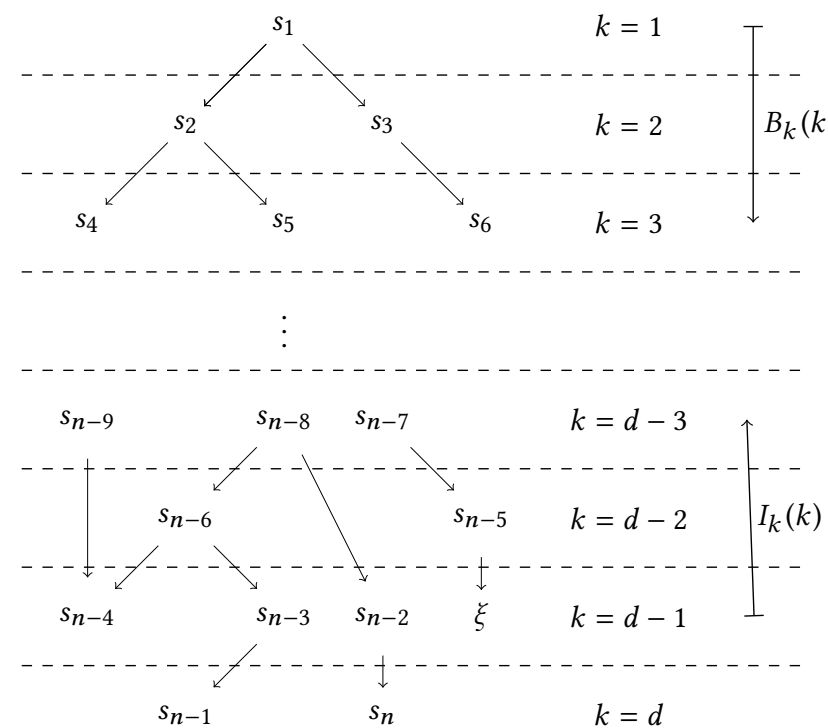

Figure 3: Visual representation of our proposed extension. Each dashed section represents the states reachable after $k$ iterations. The arrows show the "direction" of the verification by the base case $B_{k}(k)$ and the inductive step $I_{k}(k)$. The forward condition $F_{k}(k)$ is not shown in this representation but it is a forward check, similar to $B_{k}(k)$.

the inductive step, our extension selects the first state $s_{d}=\left\langle p_{c}, V_{d}\right\rangle$ and translates it into a new safety property $\varphi\left(s_{i}, s_{d}\right)$ :

$$
\varphi\left(s_{i}, s_{d}\right)=\bigwedge_{i=1}^{n} v_{1}^{i} \neq v_{1}^{d}
$$

which checks if a given state $s_{i}$ is the first state in the counterexample. Given the optimal nature of the algorithm, this is sufficient to find the property violation. Our algorithm then defines a new base case step $B^{\prime}\left(k, s_{d}\right)$ :

$$
\exists s_{1} \ldots s_{k} \cdot I\left(s_{1}\right) \wedge \bigwedge_{i=1}^{k-1} T\left(s_{i}, s_{i+1}\right) \wedge \bigvee_{i=1}^{k} \neg\left(\phi\left(s_{i}\right) \wedge \varphi\left(s_{i}, s_{d}\right)\right) .
$$

Thus, our proposed extension checks, in the base case $B_{k}(k)$, whether we can reach any value in path $\pi_{I}$. Note that this will only be applied to assertions inside a loop, as the inductive step only overapproximates loop variables. The algorithm will still be complete and optimal and, assuming that the error state is reachable in $k$ steps from the initial state, the solution will be found in $\left\lfloor\frac{k}{2}+1\right\rfloor$, because the forward and backward searches have to go only half way. Note that this repurposes the goal of the inductive step, from proving correctness to find paths that lead to error states $\xi$.

BohsidRunnimendexdandlietion algorithm applied to the code snippet shown in Figure 4a. It requires 6 iterations to reach the assertion failure. This means that the base case $B_{k}(k)$ will be called 6 times (i.e., $k=[1 \ldots 6]$ ), thus, the forward condition $F_{k}(k)$ and the inductive step $I_{k}(k)$ will be called 5 times each (i.e., $\left.k=[1 \ldots 5]\right)$. The

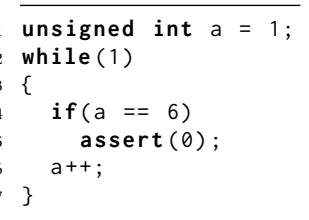

(a) Original program

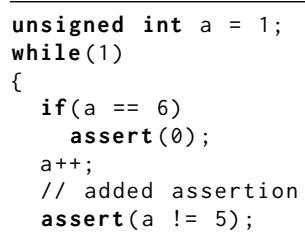

(b) Modified program

Figure 4: Code snippet example.

base case will produce for $k=6$ the counterexample $\pi_{B}=\left\langle s_{1} \rightarrow\right.$ $\langle 1, \mathrm{a}=1\rangle, s_{2} \rightarrow\langle 2, \mathrm{a}=2\rangle, s_{3} \rightarrow\langle 3, \mathrm{a}=3\rangle, s_{4} \rightarrow\langle 4, \mathrm{a}=4\rangle, s_{5} \rightarrow$ $\langle 5, \mathrm{a}=5\rangle, s_{6} \rightarrow\langle 6, \mathrm{a}=6\rangle, \xi \rightarrow\langle 7$, assert $\left.(0)\rangle\right\rangle$, which is a set of assignments that leads to an assertion failure. Now, consider the counterexample $\pi_{I}^{1}=\left\langle s_{6} \rightarrow\langle 6, \mathrm{a}=6\rangle, \xi \rightarrow\langle 7\right.$, assert $\left.(0)\rangle\right\rangle$ generated by the inductive step for $k=1$, or $\pi_{I}^{2}=\left\langle s_{5} \rightarrow\langle 5, a=5\rangle, s_{6} \rightarrow\right.$ $\langle 6, \mathrm{a}=6\rangle, \xi \rightarrow\langle 7$, assert $(0)\rangle\rangle$ for $k=2$. For $k=1$, the property violation is reachable when $a==6$, in that case, the inductive step can be interpreted as the question "is there any path of size 1 that reaches an error state?". Furthermore, each $k$ incrementation

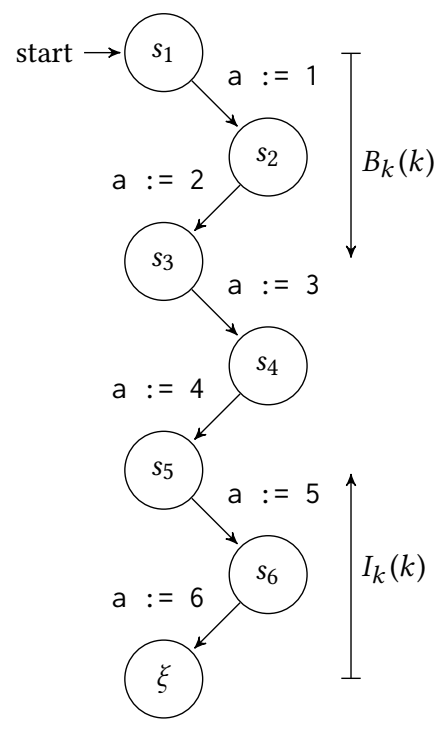

Figure 5: Unrolled CFG from code snippet in Figure $4 a$ and the "direction" of the verification for the base case $B_{k}(k)$ and the inductive step $I_{k}(k)$. ductive step produces a counterexample, our $k$-induction extension will collect the first state of this counterexample and will add a new safety property in the CFG, which will reduce the state space to be explored. 


\section{EXPERIMENTAL EVALUATION}

In order to evaluate our $k$-induction algorithm extension, we selected a number of benchmarks from the International Competition on Software Verification (SV-COMP) 2017 [1]. We compare the results from the original $k$-induction and our extended version. Currently, our extension is able to automatically identify the initial state from the counterexample generated by the inductive step, but it is unable to correctly generate and add the new verification property $\varphi\left(s_{i}, s_{d}\right)$ required by $B^{\prime}\left(k, s_{d}\right)$. As a result, the programs evaluated by our extension were manually changed to add the initial state $s_{d}$ of the counterexample generated by the inductive step. In order to establish a fair comparison, we also add that time to obtain the initial state to our extended $k$-induction. Here, we do not compare our extended $k$-induction to plain BMC since we are interested in checking the efficiency and efficacy of our new approach over the existing ESBMC $k$-induction.

Benchmark description. The benchmarks called sum $0^{*}$ are similar to the program in Figure 1, but contain a bug in different depths. The benchmarks rangesum ${ }^{*}$ check if a function is "deterministic" w.r.t. all possible permutations of an input array; the number in the benchmark name represents the size of the array. The benchmark const_false checks if a constant holds after 1024 iterations (but checks the wrong value after the iterations); diamond checks if a counter that is being nondeterministically incremented is even after 99 iterations; and Problem01_label15 is the representation of a reactive system.

Experimental setup. All experiments were conducted on a computer with an Intel Core i7-2600 running at $3.40 \mathrm{GHz}$ and $24 \mathrm{~GB}$ of RAM under Fedora 25 64-bit. We used ESBMC v4.6 [6] and no time or memory limit were set for the verification tasks.

Availability of data $\&$ tools. Our experiments are based on a set of publicly available benchmarks. All tools, benchmarks, and results of our evaluation are available on a web page. ${ }^{1}$

\begin{tabular}{|l|r||r|r|r|r|r|c|}
\hline \multirow{2}{*}{ Benchmark } & \multicolumn{1}{|c||}{} & \multicolumn{3}{|c|}{$k$-induction } & \multicolumn{3}{|c|}{ Extended $k$-induction } \\
\cline { 3 - 8 } & 19 & $T(\mathrm{~s})$ & $M(\mathrm{MB})$ & $k$ & $T(\mathrm{~s})$ & $M(\mathrm{MB})$ & $k$ \\
\hline sum04.c & 18 & $\mathbf{1}$ & 38.7 & 9 & $\mathbf{1}$ & 38.7 & 5 \\
\hline sum01.c & 25 & 3 & 38.9 & 11 & $\mathbf{1}$ & 38.8 & 6 \\
\hline sum03.c & 24 & 13 & 43.6 & 11 & $\mathbf{1}$ & 38.8 & 6 \\
\hline diamond1.c & 64 & 7 & 66.2 & 4 & $\mathbf{6}$ & 39.1 & 26 \\
\hline rangesum.c & 59 & 11 & 72.3 & 6 & $\mathbf{1}$ & 65.0 & 2 \\
\hline rangesum05.c & 59 & 28 & 78.2 & 11 & $\mathbf{1 6}$ & 47.5 & 3 \\
\hline rangesum10.c & 594 & 7 & 87.3 & 5 & $\mathbf{5}$ & 70.3 & 4 \\
\hline Problem01_label15.c & 59 & 101 & 99.9 & 21 & $\mathbf{2 6}$ & 78.2 & 12 \\
\hline rangesum20.c & 59 & 847 & 269.5 & 41 & $\mathbf{9 0}$ & 113.9 & 22 \\
\hline rangesum40.c & 20 & 2606 & 796.6 & 1025 & $\mathbf{8 9 0}$ & 253.2 & 513 \\
\hline const.c & 59 & 80272 & 1106.9 & 61 & $\mathbf{1 5 9}$ & 134.6 & 32 \\
\hline rangesum60.c & 88 & 6991 & 228.1 & 104 & $\mathbf{9 9}$ & 79.8 & 53 \\
\hline \hline Average & 1059 & 83897 & 2737.2 & 1255 & $\mathbf{1 1 9 7}$ & 957.5 & 638 \\
\hline Total & & & & & & & \\
\hline
\end{tabular}

Table 1: Preliminary evaluation over the SV-COMP 2017 benchmarks.

\subsection{Preliminary Results}

Table 1 shows the preliminary results obtained from the original $k$ induction and our proposed extension. Here, $L O C$ is the number of lines in the program, $T$ is the time needed to verify the program in seconds, $M$ is the memory used by the tools to verify the programs

\footnotetext{
${ }^{1}$ http://esbmc.org/benchmarks/fse2018.zip
}

in megabytes ${ }^{2}$ and $k$ is the number of steps needed to find the bug. The last lines show the average and cumulative numbers for each of the columns. We order the benchmarks in relation to the memory required by the original $k$-induction.

The first noticeable aspect of the results is that the time of the verification is not related to the number of steps or the program size. The closest relation between the verification time is the state space explored by each step (more specifically the inductive step), the bigger the state space, longer it will take to find a solution; this can somehow be summarized by memory used by the tool during the verification.

The evaluation for this set of benchmarks show that our extension to the $k$-induction algorithm potentially cuts the verification time considerably in cases where the state space explored is large. For small cases (e.g., the sum $0^{*} . c$ benchmarks), our extension does not slow down or uses more memory than the original $k$-induction and for large cases, the gains were substantial (e.g., the verification time of rangesum60.c was 504x faster). In terms of the steps needed to find the bug, the extended version of the $k$-induction required $\left\lfloor\frac{k}{2}+1\right\rfloor$, as expected.

We also compare the results of the extended $k$-induction with an incremental BMC approach and we observed that our extended $k$-induction is as good as an incremental BMC, in most cases. The extended $k$-induction is as fast as the incremental BMC for small bounds (and it is even faster than the incremental BMC approach in rangesum60.c), and it is not as slow as the original $k$-induction for large bounds. Our proposed approach is somewhat in the middle between the original $k$-induction and the incremental BMC, it is able to prove correctness and find bugs consuming less resources (i.e., time and memory) than the original $k$-induction but, when the program is unsafe, it is slower than the incremental BMC.

\section{RELATED WORK}

Bischoff et al. [4] propose a methodology to use BDDs and SAT solvers for the verification of programs. The BDDs are responsible for the target enlargement, collecting the under-approximate reachable state sets, followed by the SAT-based verification with the newly computed sets. The authors implemented the technique in the Intel BOolean VErifier (BOVE) and showed that the time was up to five times smaller. Compared to this work, we only use $k$-induction and SMT solvers; the inductive step in the $k$-induction algorithm is responsible for enlarging the target and the SMT solver checks for satisfiability.

Jovanović et al. [10] present a reformulation of IC3, separating the reachability checking from the inductive reasoning. They further replace the regular induction algorithm by the $k$-induction algorithm and show that it provides more concise invariants. The authors implemented the algorithm in the SALLY model checker using Yices2 to do the forward search and MathSAT5 to do the backward search. They showed that the new algorithm is able to solve a number of real-world benchmarks, at least as fast as other approaches. Compared to this work, our proposed extended $k$-induction uses consequent BMC calls to find a solution. We also implement our approach independent of solvers and it can be used

${ }^{2}$ We used the command /usr/bin/time - $v$ from linux to measure both the time and the memory usage 
with any SMT solver supported by ESBMC; however, both searches will be done with the same solver.

\section{CONCLUSION}

In this paper, our main contribution is a novel extension to the $k$-induction algorithm, to perform a bidirectional search instead of the conventional iterative deepening search. The extension is currently under development using ESBMC. We plan to evaluate the improvement over the SV-COMP benchmarks, where the original $k$-induction algorithm already proved to be the state-of-art, if compared to other $k$-induction tools [1]. The preliminary results show that the extension has the potential to substantially improve the verification time for problems with large state space, while maintaining a small verification time for small programs. In one particularly large program (in terms of state space), our extension allowed the $k$-induction algorithm to find the property violation on average using half of the steps and a fraction of the resources.

\section{REFERENCES}

[1] Dirk Beyer. 2017. Software Verification with Validation of Results (Report on SV-COMP 2017). In TACAS (LNCS), Vol. 10206. 331-349.

[2] Armin Biere. 2009. Handbook of Satisfiability. Frontiers in Artificial Intelligence and Applications, Vol. 185. IOS Press, Chapter 14, 455-481.

[3] Armin Biere, Alessandro Cimatti, Edmund Clarke, and Yunshan Zhu. 1999. Symbolic Model Checking Without BDDs. In TACAS (LNCS), Vol. 1633. 193-207.

[4] Gabriel P. Bischoff, Karl S. Brace, G. Cabodi, and S. Nocco, S.and Quer. 2005 Exploiting Target Enlargement and Dynamic Abstraction within Mixed BDD and SAT Invariant Checking. Electronic Notes in Theoretical Computer Science 119,2 (2005), 33-49.

[5] Edmund Clarke, Daniel Kroening, and Flavio Lerda. 2004. A Tool for Checking ANSI-C Programs. In TACAS (LNCS), Vol. 2988. 168-176.

[6] Lucas C. Cordeiro, Bernd Fischer, and João Marques-Silva. 2012. SMT-Based Bounded Model Checking for Embedded ANSI-C Software. IEEE Transactions on Software Engineering 38, 4 (2012), 957-974.

[7] Zakir Durumeric, James Kasten, David Adrian, J. Alex Halderman, Michael Bailey, Frank Li, Nicolas Weaver, Johanna Amann, Jethro Beekman, Mathias Payer, and Vern Paxson. 2014. The Matter of Heartbleed. In IMC. 475-488.

[8] Mikhail Y. R. Gadelha, Hussama I. Ismail, and Lucas C. Cordeiro. 2017. Handling loops in bounded model checking of C programs via k-induction. STTT 19, 1 (2017), 97-114.

[9] Steve Heath. 2003. Embedded Systems Design. Newnes, Oxford, United Kingdom. 430 pages.

[10] Dejan Jovanović and Bruno Dutertre. 2016. Property-directed K-induction. In FMCAD. 85-92.

[11] Daniel Kroening, Joël Ouaknine, Ofer Strichman, Thomas Wahl, and James Worrell. 2011. Linear Completeness Thresholds for Bounded Model Checking. In CAV (LNCS), Vol. 6806. 557-572.

[12] Daniel Kroening, Joël Ouaknine, Ofer Strichman, Thomas Wahl, and James Worrell. 2011. Linear Completeness Thresholds for Bounded Model Checking. In CAV (LNCS), Vol. 6806. 557-572. https://doi.org/10.1007/978-3-642-22110-1 44

[13] Florian Merz, Stephan Falke, and Carsten Sinz. 2012. LLBMC: Bounded Model Checking of C and C++ Programs Using a Compiler IR. In VSTTE (LNCS), Vol. 7152. 146-161.

[14] Felipe R. Monteiro, Erickson H. da S. Alves, Isabela S. Silva, Hussama I. Ismail, Lucas C. Cordeiro, and Eddie B. de Lima Filho. 2018. ESBMC-GPU A ContextBounded Model Checking Tool to Verify CUDA Programs. Science of Computer Programming 152 (2018), 63 - 69.

[15] Shaz Qadeer and Jakob Rehof. 2005. Context-Bounded Model Checking of Concurrent Software. In TACAS (LNCS), Vol. 3440. 93-107.

[16] Mary Sheeran, Satnam Singh, and Gunnar Stålmarck. 2000. Checking Safety Properties Using Induction and a SAT-Solver. In FMCAD. 108-125. 